\title{
Universal shape ratios for polymers grafted at a flat surface
}

\author{
Maria Serena Causo \\ INFM-NEST and Scuola Normale Superiore \\ Piazza dei Cavalieri, 7 \\ I-56100 Pisa, ITALY \\ Internet: causo@sns.it
}

July 24, 2013

\begin{abstract}
We consider dilute non-adsorbed polymers grafted at an impenetrable surface and compute several quantities which characterize the polymer shape: the asphericity and the ratios of the eigenvalues of the radius-of-gyration tensor. The results are only slightly different from those obtained for polymers in the bulk, showing that the surface has little influence on the polymer shape.
\end{abstract}




\section{Introduction}

It has been known since 1934 that polymers are not istantaneously spherical in shape [1]. Since then a lot of work has been devoted to quantify the average deviation from spherical symmetric shape in the limit of large degree of polymerization, since this deviation plays an important role in the hydrodynamic behaviour of dilute polymer solutions [2]. There are several observables which quantify the shape of polymers. One considers the (squared) radius-of-gyration tensor and its eigenvalues $q_{1}, \ldots, q_{d}$, which give the square lengths of the polymer along the principal axes of inertia [3]. The ratios $\left\langle q_{i}\right\rangle /\left\langle q_{j}\right\rangle$ or, alternatively, $\left\langle q_{i} / q_{j}\right\rangle$ are universal and may be used to give a universal characterization of the polymer shape. Alternatively, one may use the shape asphericity that allows to distinguish between spherical and rodlike configurations. The equivalent ellipsoid has been experimentally determined in a small-angle x-ray scattering experiment for globular proteins [4].

The shape of Gaussian polymers has been extensively studied. The shape asphericity, which in its two variants is proportional to the istantaneous variance of the average of eigenvalues of the radius-of-gyration tensor, has been exactly computed [5, 6]. Instead, the ratios of the eigenvalues $q_{i}$ are not exactly known. There are perturbative estimates at second order in $1 / d$ [7], which are, however, not very predictive in the physically relevant case $d=3$, and several estimates from numerical simulations [7] [13]. The numerical estimates are in good agreement with each other.

In the case of polymers with excluded-volume interactions, asphericity has been computed to second order in $\epsilon=4-d$ in Ref. [14] and by numerical simulations in Refs. [9, 14] 22], while the ratios of the eigenvalues of the radius-of-gyration tensor have been computed by Monte Carlo simulations in Refs. 8, 10, 18 22]. Here we consider the case of polymers with excluded-volume interactions grafted at an impenetrable interface at the ordinary transition 23 25]. Polymers are modeled as $N$-step self-avoiding walks on a simple cubic lattice. We perform an extensive Monte Carlo simulation using a variant of the pivot algorithm 26 28], the cut-and-permute algorithm, which has been shown to be optimal for non-adsorbed polymers grafted at a surface [29]. The efficiency of the algorithm allows us to simulate long walks with $N \leq 32000$ and to study how the presence of the surface changes the shape of the polymer.

We find that the main effect of the presence of the surface is to increase the length of the longest axis of the inertia ellipsoid with respect to the other two, whose relative length remains, instead, of the same order. Nevertheless, being this increment in asphericity only of a few percents, we expect that it can only be detected in highresolution experiments. The paper is organized as follows. In Sec. 2 we define the observables that will be computed. In Sec. 3 we present our Monte Carlo results, paying due attention to the corrections to scaling. In Sec. 4 we compare our results with those for polymers in the bulk. 


\section{Definitions}

We model the polymer as an $N$-step self-avoiding walk on a simple cubic lattice, each step representing a single monomer. The polymer has one of the two endpoints grafted at an impenetrable flat surface of equation $z=0$, so that the accessible space is $\mathbb{R}_{+}^{3}=\left\{\mathbf{r}=\left(\mathbf{r}_{\|}, z\right): \mathbf{r}_{\|} \in \mathbb{R}^{2}, z \geq 0\right\}$. Let us denote by $\omega=\left\{\omega_{0}, \ldots, \omega_{N}\right\}$ a generic polymer configuration, where $\omega_{i}=\left(\omega_{i}^{1}, \omega_{i}^{2}, \omega_{i}^{3}\right) \in \mathbb{R}_{+}^{3}$ is the position vector of the $i$-th monomer and $\omega_{0}^{3}=0$, and let us call $W_{N}$ the set of allowed configurations.

One can introduce several invariant quantities which describe the shape of the polymer. In generic dimension $d$ we define the radius-of-gyration or shape tensor

$$
Q_{\alpha \beta}=\frac{1}{N} \sum_{i>j}\left(\omega_{i}^{\alpha}-\omega_{j}^{\alpha}\right)\left(\omega_{i}^{\beta}-\omega_{j}^{\beta}\right),
$$

whose trace concides with the squared radius of gyration

$$
R_{g}^{2}=\operatorname{Tr} Q
$$

If we denote with $\left\{q_{1}, q_{2}, \ldots, q_{d}\right\}$ the set of eigenvalues of $Q$, ordered in such a way that $q_{1} \leq q_{2} \leq \ldots \leq q_{d}$, and with $\bar{q}=\frac{1}{d} \operatorname{Tr} Q$ their average, we can define the shape asphericity of the polymer as

$$
A_{d}(\omega)=\frac{1}{d(d-1)} \sum_{\alpha=1}^{d} \frac{\left(q_{\alpha}-\bar{q}\right)^{2}}{\bar{q}^{2}}=\frac{d}{d-1} \frac{\operatorname{Tr}\left(\widehat{Q}_{d}^{2}\right)}{(\operatorname{Tr} Q)^{2}},
$$

where $\widehat{Q}=Q-\frac{1}{d}(\operatorname{Tr} Q) I_{d}$ and $I_{d}$ is the identity matrix. Since the eigenvalues of $Q$ correspond to the squared lengths of the equivalent ellipsoid axes and the eigenvectors represent the axes directions, $A_{d}(\omega)=0$ for a spherically symmetric configurationin this case all eigenvalues take the same value - while $A_{d}(\omega)=1$ if the polymer is completely elongated and all eigenvalues except one vanish. The mean asphericity is obtained by averaging $A_{d}(\omega)$ over the allowed polymer conformations

$$
A_{d}^{N}=\frac{1}{c_{N}} \sum_{\omega \in W_{N}} A_{d}(\omega)
$$

where $c_{N}$ is the number of allowed configurations and behaves as $c_{N} \sim \mu^{N} N^{\gamma_{1}-1}$ in the critical limit $N \rightarrow \infty$. Here $\mu$ is the critical fugacity, which does not depend on the presence of the surface, and $\gamma_{1}$ is the susceptibility critical surface exponent $\gamma_{1}=0.679(2)$ (Ref. [30]), $\gamma_{1} \approx 0.680$ (Ref. [31]). Other invariant quantities are often considered in order to characterize the polymer shape. For instance, one may consider

$$
\widehat{A}_{d}^{N}=\frac{d}{d-1} \frac{\left\langle\operatorname{Tr}\left(\widehat{Q}_{d}^{2}\right)\right\rangle}{\left\langle(\operatorname{Tr} Q)^{2}\right\rangle},
$$

or the relative magnitudes of the averages of the eigenvalues, $\left\langle q_{2}\right\rangle /\left\langle q_{1}\right\rangle,\left\langle q_{3}\right\rangle /\left\langle q_{1}\right\rangle$. The advantage of studying these quantities is that they do not require computing an average of the ratio of highly fluctuating quantities. 
In the limit of large degree of polymerization $N \rightarrow \infty$, these quantities reach universal values with corrections of order $N^{-\Delta}$,

$$
\begin{aligned}
A_{d}^{N} & =A_{d}\left(1+\frac{B}{N^{\Delta}}+\ldots\right), \\
\widehat{A}_{d}^{N} & =\widehat{A}_{d}\left(1+\frac{\widehat{B}}{N^{\Delta}}+\ldots\right), \\
\frac{\left\langle q_{2}^{N}\right\rangle}{\left\langle q_{1}^{N}\right\rangle} & =L_{2,1}^{2}\left(1+\frac{l_{2,1}}{N^{\Delta}}+\ldots\right), \\
\frac{\left\langle q_{3}^{N}\right\rangle}{\left\langle q_{1}^{N}\right\rangle} & =L_{3,1}^{2}\left(1+\frac{l_{3,1}}{N^{\Delta}}+\ldots\right),
\end{aligned}
$$

where $\Delta$ is the leading correction-to-scaling exponent. On the other hand, the eigenvalues of the gyration tensor scale as

$$
\left\langle q_{i}^{N}\right\rangle=b_{i} N^{2 \nu}\left(1+\frac{c_{i}}{N^{\Delta}}+\cdots\right) \quad i=1,2,3
$$

In the following section we present a Monte Carlo study of the universal behaviour of these quantities.

\section{Monte Carlo study of the shape asphericity}

We computed the eigenvalues of the shape tensor and the asphericities by Monte Carlo simulations. The system was modeled as a self-avoiding walk grafted at the origin of the half cubic lattice $\mathbb{Z}_{+}^{3}$. In order to sample the phase space $W_{N}$, we used a nonlocal Monte Carlo algorithm, the cut-and-permute algorithm [29]. It is an optimal algorithm for studying polymers grafted at an impenetrable surface at the ordinary transition, since autocorrelation times in CPU units for global observables are simply proportional to the degree of polymerization $N$. The algorithm has, therefore, the same optimal behaviour as the pivot algorithm for polymers in the bulk.

The system in the bulk is known to have strong corrections to scaling [32], since the leading correction-to-scaling exponent is $\Delta_{\text {bulk }} \approx 0.5$. It is expected that polymers in the presence of a surface show analogous corrections, and, indeed, our estimates of the leading correction-to-scaling exponent are close to 0.5.

Since the presence of large corrections to scaling introduces systematic errors in the estimates for finite $N$, we studied the system for large $N, 1000 \leq N \leq 32000$. At first, we studied the behaviour of the eigenvalues of the shape tensor and of its trace $R_{g}^{2}$. The results are displayed in Table 1. These quantities are expected to increase as $N^{2 \nu}$, being $\nu$ the same critical exponent as in the bulk. In order to check this prediction, we performed fits of the form

$$
\begin{aligned}
\left\langle q_{i}\right\rangle_{N} & =b_{i} N^{2 \nu} \quad i=1,2,3, \\
\left\langle R_{g}^{2}\right\rangle & =b N^{2 \nu}
\end{aligned}
$$


where $b, b_{i}$, and $\nu$ are free parameters. In Eq. (11) corrections to scaling are neglected. We expect, therefore, a systematic error in our estimates. In order to take into account the effect of corrections to scaling, we have performed different fits, considering only data with $N \geq N_{\text {min }}$. The effective exponents which are obtained decrease with increasing $N_{\min }$ for all values of $N_{\text {min }}$ considered, so that we can only give an upper bound for the exponent $\nu$. The estimates which are less affected by corrections to scaling appear to be those corresponding to the largest eigenvalue. Therefore, taking as an upper bound for $\nu$ the estimate from the fit of $\left\langle q_{3}\right\rangle$ with $N_{\min }=16000$, we obtain $\nu \leq 0.58774(35)$, which agrees with the best estimates obtained so far for polymers in the bulk, which are $\nu=0.58758(7)$ (Ref. 34]), $\nu=0.5877(6)$ (Ref. 35]).

Let us consider now the ratios of eigenvalues. For such invariant quantities we performed the following three-parameter fits

$$
\frac{\left\langle q_{k}\right\rangle_{N}}{\left\langle q_{1}\right\rangle_{N}}=L_{k, 1}^{2}+D\left(\frac{N_{0}}{N}\right)^{\Delta} \quad k=1,2,
$$

where $L_{k, 1}^{2}, D$, and $\Delta$ are free parameters and $N_{0}=16000$ is a fixed parameter introduced in order to reduce the cross-correlations between $D$ and $\Delta$ in the correctionto-scaling term. Again, since we are neglecting higher-order corrections to scaling, the estimates of the universal amplitudes are affected by systematic errors. In order to take into account this effect, we performed fits in which only data with $N \geq N_{\text {min }}$ were considered and we studied how our estimates approach the asymptotic value as $N_{\text {min }}$ increases. The results of the fits are shown in Table 2. In this case the estimates from the three-parameter fits appear to be very stable, so that we can choose as best estimates of the universal ratios the ones for $N_{\min }=1000: L_{2,1}^{2}=2.9272(28)$, $L_{3,1}^{2}=14.6293(66)$.

Nevertheless, one can notice that, while the correction-to-scaling exponent determined from the ratio $\left\langle q_{3}\right\rangle_{N} /\left\langle q_{1}\right\rangle_{N}$ is $\Delta=0.450(46)$, which is in agreement with the estimates for the leading correction-to-scaling exponent for polymers in the bulk [32], the estimate of $\Delta$ obtained in fits of $\left\langle q_{2}\right\rangle_{N} /\left\langle q_{1}\right\rangle_{N}$ is considerably smaller. This can be due to the presence of corrections with opposite sign and could affect our final estimate. For this reason, we also performed fits in which the leading correction-toscaling exponent was fixed at $\Delta=0.5$, and higher-order corrections were neglected. The estimates obtained are shown in the last three rows of Table 2. Estimates for $L_{2,1}^{2}$ are stable for $N_{\min }=4000$, while those for $L_{3,1}^{2}$ are already stable for $N_{\min }=1000$. In all cases the $\chi^{2}$ per degree of freedom is less than 1 . Therefore, we can take as final estimates

$$
\begin{aligned}
& L_{2,1}^{2}=2.94931(99) \\
& L_{3,1}^{2}=14.6449(60) .
\end{aligned}
$$

One can notice that the estimate in Eq. (14) agrees with the one obtained without fixing $\Delta$, while $L_{2,1}^{2}$ in Eq. (13) is not compatible with the previous estimate. Since we know a priori that $\Delta$ should be approximately $1 / 2$, we are inclined to consider (14) a more reliable estimate. 
We performed the same kind of analysis for the asphericities, performing fits of the form

$$
\begin{aligned}
& A_{3}^{N}=A_{3}+D\left(\frac{N_{0}}{N}\right)^{\Delta}, \\
& \widehat{A}_{3}^{N}=\widehat{A}_{3}+\widehat{D}\left(\frac{N_{0}}{N}\right)^{\Delta} .
\end{aligned}
$$

The results are reported in Table 3 . Fits are not very stable and the amplitude appearing in the correction to scaling is very small. For $N_{\text {min }}=1000$ we estimated $D \approx(47 \pm 10) \times 10^{-5}$ and $\widehat{D} \approx(43 \pm 7) \times 10^{-5}$. As before, we also performed a two-parameter fit in which the leading correction-to-scaling exponent was fixed at $\Delta=0.5$. Since all estimates for $N_{\min } \geq 1000$ are compatible within error bars, our final estimates are

$$
\begin{aligned}
& A_{3}=0.44520(15) \\
& \widehat{A}_{3}=0.55949(13) .
\end{aligned}
$$

These estimates are in agreement with those obtained letting $\Delta$ be a free parameter.

\section{Comparison of the results with the case of poly- mers in the bulk}

Let us compare our results with the ones for polymers in the bulk [8 10, 14, 18 22]. An early Monte Carlo simulation [14] of polymers with length $N \leq 220$, in which the presence of corrections to scaling was taken into account, gave $A_{3}=0.431(2)$. Since then, more computing power and more efficient algorithms became available and much longer polymers could be studied. In Ref. [19], self-avoiding walks for a single value of $N(N=963)$ were simulated using the pivot algorithm, obtaining $A_{3}=0.43333(8)$ and $\widehat{A}_{3}=0.54722(9)$ for the shape asphericities. From the data in Ref. [19] for the shape factors, we can compute the eigenvalue ratios $L_{3,1}^{2}=14.1102(52)$ and $L_{2,1}^{2}=2.9631(12)$. Since only one value of $N$ was considered, no analysis of the corrections to scaling could be done. Thus, we expect the errors to be grossly underestimated. Indeed, our results show that, for $N \approx 10^{3}$, the systematic error due to the scaling corrections is much larger than the quoted statistical error. In Refs. 21, 22] even longer walks were considered and data were extrapolated to infinite length with leading correction-to-scaling exponents $\Delta=0.5$ and $\Delta=0.46$. The estimates for the asphericities are: $A_{3}=0.4302$ and $\widehat{A}_{3}=0.5450$ for $\Delta=0.5 ; A_{3}=0.4303$ and $\widehat{A}_{3}=0.5452$ for $\Delta=0.46$. From the asymptotic values for shape factors, we obtain for the eigenvalue ratios: $L_{3,1}^{2}=13.89, L_{2,1}^{2}=2.934$ for $\Delta=0.5$; and $L_{3,1}^{2}=13.92$ $L_{2,1}^{2}=2.939$ for $\Delta=0.46$. No error bars on the extrapolated values were reported in Refs. [21, 22]. The asphericity $A_{3}$ has also been computed in an extensive Monte Carlo simulation in Ref. [18], where walks of length $N \geq 40000$ were considered. If 


\begin{tabular}{|c||c|c|c|c|}
\hline \hline$N$ & $\left\langle q_{1}\right\rangle_{N} \pm \delta\left\langle q_{1}\right\rangle_{N}$ & $\nu \pm \delta \nu$ & $\chi^{2}$ & DF \\
\hline 1000 & $35.613 \pm 0.030$ & $0.59000 \pm 0.00009$ & 153.355 & 3 \\
4000 & $184.151 \pm 0.058$ & $0.58949 \pm 0.00010$ & 41.982 & 2 \\
8000 & $417.76 \pm 0.057$ & $0.58901 \pm 0.00013$ & 1.294 & 1 \\
16000 & $945.49 \pm 0.248$ & $0.58867 \pm 0.00033$ & 0 & 0 \\
32000 & $2138.31 \pm 0.818$ & & & \\
\hline \hline$N$ & $\left\langle q_{2}\right\rangle_{N} \pm \delta\left\langle q_{2}\right\rangle_{N}$ & $\nu \pm \delta \nu$ & $\chi^{2}$ & DF \\
\hline 1000 & $105.71 \pm 0.10$ & $0.58936 \pm 0.00010$ & 77.460 & 3 \\
4000 & $545.34 \pm 0.18$ & $0.58894 \pm 0.00011$ & 20.057 & 2 \\
8000 & $1235.79 \pm 0.38$ & $0.58834 \pm 0.00018$ & 1.348 & 1 \\
16000 & $2794.68 \pm 0.78$ & $0.58799 \pm 0.00035$ & 0 & 0 \\
32000 & $6314.5 \pm 2.5$ & & & \\
\hline \hline \hline$N$ & $\left\langle q_{3}\right\rangle_{N} \pm \delta\left\langle q_{3}\right\rangle_{N}$ & $\nu \pm \delta \nu$ & $\chi^{2}$ & DF \\
\hline 1000 & $528.12 \pm 0.59$ & $0.58881 \pm 0.00011$ & 30.2 & 3 \\
4000 & $2714.9 \pm 1.0$ & $0.58853 \pm 0.00013$ & 8.27 & 2 \\
8000 & $6145.0 \pm 2.1$ & $0.58814 \pm 0.00020$ & 1.44 & 1 \\
16000 & $13893.5 \pm 4.3$ & $0.58774 \pm 0.00038$ & 0 & 0 \\
32000 & $31381 . \pm 13$. & & & \\
\hline \hline$N$ & $\left\langle R_{g}^{2}\right\rangle \pm \delta\left\langle R_{g}^{2}\right\rangle$ & $\nu \pm \delta \nu$ & $\chi^{2}$ & DF \\
\hline 1000 & $670.51 \pm 0.36$ & $0.58918 \pm 0.00009$ & 44.6 & 3 \\
4000 & $3445.4 \pm 1.3$ & $0.58857 \pm 0.00013$ & 4.13 & 2 \\
8000 & $7796.6 \pm 2.4$ & $0.58830 \pm 0.00020$ & 0.69 & 1 \\
16000 & $17628.2 \pm 4.8$ & $0.58801 \pm 0.00040$ & 0 & 0 \\
32000 & $39831 \pm 19$ & & & \\
\hline
\end{tabular}

Table 1: Monte Carlo results for the eigenvalues and for the trace of the radius-ofgyration tensor $Q$ and effective exponents computed using the results for walks of length $\geq N_{\min }=N$. Data are fitted as in Eq. (11). "DF" is the number of degrees of freedom. 


\begin{tabular}{|c|c|c|c|c|c|c|}
\hline$N$ & \multicolumn{3}{|c|}{$\frac{\left\langle q_{2}\right\rangle_{N}}{\left\langle q_{1}\right\rangle_{N}} \pm \delta \frac{\left\langle q_{2}\right\rangle_{N}}{\left\langle q_{1}\right\rangle_{N}}$} & \multicolumn{3}{|c|}{$\frac{\left\langle q_{3}\right\rangle_{N}}{\left\langle q_{1}\right\rangle_{N}} \pm \delta \frac{\left\langle q_{3}\right\rangle_{N}}{\left\langle q_{1}\right\rangle_{N}}$} \\
\hline 1000 & \multicolumn{3}{|c|}{$2.9683 \pm 0.0025$} & \multicolumn{3}{|c|}{$14.830 \pm 0.018$} \\
\hline 4000 & \multicolumn{3}{|c|}{$2.96137 \pm 0.00073$} & \multicolumn{3}{|c|}{$14.7426 \pm 0.0052$} \\
\hline 8000 & \multicolumn{3}{|c|}{$2.95804 \pm 0.00063$} & \multicolumn{3}{|c|}{$14.7101 \pm 0.0047$} \\
\hline 16000 & \multirow{2}{*}{\multicolumn{3}{|c|}{$\begin{array}{l}2.95580 \pm 0.00055 \\
2.95303 \pm 0.00078\end{array}$}} & \multicolumn{3}{|c|}{$14.6945 \pm 0.0040$} \\
\hline 32000 & & & & \multicolumn{3}{|c|}{$14.6762 \pm 0.0057$} \\
\hline 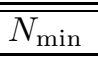 & $\overline{L_{2,1}^{2} \pm \delta L_{2,1}^{2}}$ & $\overline{\Delta \Delta \pm \delta \Delta}$ & $\overline{\chi^{2} / D F}$ & $\overline{L_{3,1}^{2} \pm \delta L_{3,1}^{2}}$ & $\overline{\Delta \Delta \pm \delta \Delta}$ & $\overline{\chi^{2} / D F}$ \\
\hline 1000 & $2.9272 \pm 0.0028$ & $0.150 \pm 0.017$ & "0.167 & $\overline{14.6293 \pm 0.0066}$ & 0.450土0.046 & 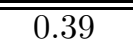 \\
\hline 4000 & $2.9273 \pm 0.0032$ & $0.15 \pm 0.020$ & 0.33 & $14.6415 \pm 0.0074$ & $0.525 \pm 0.083$ & 0.73 \\
\hline$N_{\min }$ & $\overline{L_{2,1}^{2} \pm \delta L_{2,1}^{2}}$ & $\Delta=0.5$ & $\overline{\chi^{2} / D F}$ & $\overline{L_{3,1}^{2} \pm \delta L_{3,1}^{2}}$ & $\overline{\Delta \Delta=0.5}$ & $\overline{\chi^{2} / D F}$ \\
\hline 1000 & $2.95026 \pm 0.00082$ & & 1.36 & $\overline{14.6449 \pm 0.0060}$ & & 0.32 \\
\hline 4000 & $2.94931 \pm 0.00099$ & & 0.56 & $14.6431 \pm 0.0072$ & & 0.37 \\
\hline 8000 & $2.9487 \pm 0.0015$ & & 0.82 & $14.646 \pm 0.011$ & & 0.56 \\
\hline
\end{tabular}

Table 2: Monte Carlo results for the ratios of eigenvalues and results of the corresponding fits for different values of $N_{\text {min }}$. Data were fitted to the scaling form (12). In the first set of fits $\Delta$ was taken as a free parameter, while in the second set $\Delta=0.5$.

\begin{tabular}{|c|c|c|c|c|c|c|}
\hline$\overline{N N}$ & \multicolumn{3}{|c|}{ 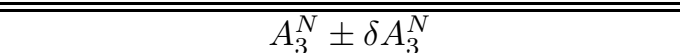 } & \multicolumn{3}{|c|}{$\widehat{\widehat{A}} \widehat{A}_{3}^{N} \pm \delta \widehat{A_{3}^{N}}$} \\
\hline 1000 & \multicolumn{3}{|c|}{$0.44806 \pm 0.00033$} & \multicolumn{3}{|c|}{$0.56210 \pm 0.00040$} \\
\hline 4000 & \multicolumn{3}{|c|}{$0.44641 \pm 0.00017$} & \multicolumn{3}{|c|}{$0.56104 \pm 0.00011$} \\
\hline 8000 & \multicolumn{3}{|c|}{$0.44623 \pm 0.00018$} & \multicolumn{3}{|c|}{$0.56035 \pm 0.00010$} \\
\hline$\overline{16000}$ & \multicolumn{3}{|c|}{$0.44604 \pm 0.00013$} & \multicolumn{3}{|c|}{$0.56010 \pm 0.00008$} \\
\hline 32000 & \multicolumn{3}{|c|}{$0.44554 \pm 0.00016$} & \multicolumn{3}{|c|}{$0.56019 \pm 0.00012$} \\
\hline$N_{\min }$ & $A_{3} \pm \delta A_{3}$ & $\overline{\Delta \Delta \pm \delta \Delta}$ & $\overline{\chi^{2} / D F}$ & $\widehat{A_{3} \pm \delta \widehat{A}_{3}}$ & $\overline{\Delta \Delta \pm \delta \Delta}$ & $\overline{\chi^{2} / D F}$ \\
\hline 1000 & $0.44520 \pm 0.00015$ & $0.538 \pm 0.085$ & 1.70 & $0.5596 \pm 0.00011$ & $0.575 \pm 0.080$ & 3.781 \\
\hline$N_{\min }$ & $A_{3} \pm \delta A_{3}$ & $\overline{\Delta \Delta=0.5}$ & $\overline{\chi^{2} / D F}$ & $\widehat{A_{3} \pm \delta \widehat{A}_{3}}$ & $\overline{\Delta \Delta=0.5}$ & $\overline{\chi^{2} / D F}$ \\
\hline 1000 & $0.44520 \pm 0.00015$ & & 1.12 & $0.55949 \pm 0.00013$ & & 2.58 \\
\hline 4000 & $0.44530 \pm 0.00022$ & & 1.50 & $0.55945 \pm 0.00015$ & & 3.72 \\
\hline 8000 & $0.44496 \pm 0.00035$ & & 1.46 & $0.55989 \pm 0.00024$ & & 1.67 \\
\hline
\end{tabular}

Table 3: Monte Carlo results for asphericities and results of the corresponding fits for different $N_{\text {min }}$. Data were fitted to the scaling form (16). In the first set of fits $\Delta$ was taken as a free parameter, while in the second set $\Delta=0.5$. 
the data reported there are fitted assuming $\Delta=0.5$, one finds $A_{3}=0.43074(57)$, which agrees within error bars with the estimate for $N=40000, A_{3}=0.4309(17)$, and with the above-reported estimates of Refs. [21,22]. The same analysis cannot be performed for the eigenvalue ratios, since the estimates at different $N$ are not available. Nevertheless, using Table 2 of Ref. [18], we obtain $L_{3,1}^{2} \approx 14.05, L_{2,1}^{2} \approx 2.963$. Both values are slightly higher than the corresponding ones in Refs. [21, 22].

Comparing our estimates (17), (18), with those reported above, we find that, due to the presence of the surface, $A_{3}$ approximately increases by $3.5 \%$, while $\widehat{A}_{3}$ by $2.6 \%$. The change in the two asphericity observables is, therefore, of the same order.

Moreover, while $L_{2,1}^{2}$ does not appreciably change, $L_{3,1}^{2}$ increases by about $5 \%$. This shows that the change in asphericity is mainly due to the fact that the length of the longest axis of the inertia ellipsoid increases with respect to the other two. This is a signal of the fact that the presence of the surface gives the polymer directionality along the normal direction to the surface, which we expect, on average, to coincide with the direction of the larger axis in the inertia ellipsoid. Nevertheless, the difference is only of a few percents. Therefore, we expect that it can be experimentally detected only in high-precision experiments.

\section{References}

[1] W. Kuhn, Kolloid Z. 68, 2 (1934).

[2] H. A. Kramers, J. Chem. Phys. 14, 415 (1946).

[3] K. Sŏlc and W. H. Stockmayer, J. Chem. Phys. 54, 2756 (1971).

[4] J. J. Müller and H. Schrauber, J. Appl. Cryst. 25, 181 (1992).

[5] J. Rudnick and G. Gaspari, J. Phys. A 19, L191 (1986).

[6] H. W. Diehl and E. Eisenriegler, J. Phys. A 22, L87 (1989).

[7] A. Beldjenna, J. Rudnick, and G. Gaspari, J. Phys. A 24, 2131 (1991).

[8] J. Mazur, C. M. Guttman, and F. McCrackin, Macromolecules 6,872 (1973).

[9] M. Bishop and J. H. Clarke, J. Chem. Phys. 90, 6647 (1989).

[10] W. Bruns, Makromol. Chem. Theory Simul. 1, 287 (1992).

[11] S. J. Sciutto, J. Phys. A 27, 7015 (1994).

[12] G. Zifferer and O. F. Olaj, J. Chem. Phys. 100, 636 (1994).

[13] S. J. Sciutto, J. Phys. A 28, 3667 (1995).

[14] O. Jagodzinski, E. Eisenriegler, and K. Kremer, J. Physique I 2, 2243 (1992).

[15] M. Bishop and C. J. Saltiel, J. Chem. Phys. 88, 6594 (1988). 
[16] J. Batoulis and K. Kremer, Macromolecules 22, 4277 (1989).

[17] J. W. Cannon, J.A. Aronovitz, and P. Goldbart, J. Physique I 1, 629 (1991).

[18] S. J. Sciutto, J. Phys. A 29, 5455 (1996).

[19] G. Zifferer, J. Chem. Phys. 109, 3691 (1998).

[20] E. G. Timoshenko, Y. A. Kuznetsov, and R. Connolly, J. Chem. Phys. 116, 3905 (2002).

[21] G. Zifferer, Macromol. Theory Simul. 8, 433 (1999).

[22] G. Zifferer and W. Preusser, Macromol. Theory Simul. 10, 397 (2001).

[23] H. W. Diehl, in Phase Transitions and Critical Phenomena, edited by C. Domb and J. L. Lebowitz (Academic, London, 1986), Vol. 10, p. 75.

[24] H. W. Diehl, Int. J. Mod. Phys. B 11, 3503 (1997).

[25] E. Eisenriegler, "Polymers near surfaces", (World Scientific, Singapore) 1993.

[26] M. Lal, Mol. Phys. 17, 1969.

[27] B. MacDonald, N. Jan, D. L. Hunter, and M. O. Steinitz, J. Phys. A 18, 2627 (1985).

[28] N. Madras and A. D. Sokal, J. Stat. Phys. 50,109 (1988).

[29] M. S. Causo, "Cut-and-permute algorithm for self-avoiding walks in the presence of surfaces", to appear in J. Stat. Phys., cond-mat/0103415.

[30] R. Hegger and P. Grassberger, J. Phys. A 27, 4096 (1994).

[31] H.W. Diehl and M. Shpot, Nucl. Phys. B 528, 595 (1998).

[32] The most precise Monte Carlo estimate of $\Delta$ is $\Delta=0.517 \pm 0.007+0.010$ [P. Belohorec and B. G. Nickel, Accurate universal and two-parameter model results from a Monte-Carlo renormalization group study, Guelph university report (September 1997)], while perturbative field theory gives a lower estimate: $\Delta=0.478 \pm 0.010$ ( $g$-expansion) and $\Delta=0.486 \pm 0.016$ ( $\epsilon$ expansion $)[R$. Guida and J. Zinn-Justin, J. Phys. A 31, 8103 (1998)].

[33] N. Madras and A. D. Sokal, J. Stat. Phys. 50, 109 (1988).

[34] P. Belohorec and B. G. Nickel, Accurate universal and two-parameter model results from a Monte-Carlo renormalization group study, Guelph university report (September 1997).

[35] B. Li, N. Madras and A. D. Sokal, J. Stat. Phys. 80, 661 (1995). 